\title{
Synaptic responses to concerted genomic evolution in Lathyrus. I. Intergenomic effects
}

\author{
A. J. WALLACE* \& R. S. CALLOW $\dagger$ \\ Plant Science and Cytogenetics Group, Department of Cell and Structural Biology, Williamson Building, The University, \\ Manchester M13 9PL, U.K.
}

\begin{abstract}
$\mathrm{C}_{0}$ autotetraploids have been derived from seven diploid $(x=7)$ species of Lathyrus, varying in genome size (11-20 pg DNA/2C) and content of moderately repetitive DNA $(5-12 \mathrm{pg} / 2 \mathrm{C})$. Pairing patterns at metaphase-I in 942 pollen mother cells (PMCs), each with a minimum of 28 chiasmata, were compared with a model relating pairing autonomy to genomic organization. Autotetraploids of one species, $L$. aphaca, form on average one multivalent more than those of the other $\operatorname{six}(5.2 \mathrm{vs}$. $3.7-4.2 / \mathrm{PMC}$ ). With the exception of $L$. aphaca, intergenomic differences in bivalent frequency and incidence of synaptic exchange correlate with the size of the genome, particularly that of the moderately repetitive component. Such nucleotypic correlations appear to reflect losses in numbers of autonomous synaptic sites, concomitant with concerted reductions in the size of the genome. They also indicate that autonomous synapsis may be governed by moderately repetitive sequences.
\end{abstract}

Keywords: Autopolyploidy, genomic evolution, Lathyrus, meiosis, multivalents, synapsis.

\section{Introduction}

Eukaryotic organisms are distinguished from prokaryotes by greater complexity, larger genomes and predominantly sexual modes of reproduction. Although the size of the genome bears some relation to the level of organization in prokaryotes, it is an evolutionary maverick in eukaryotes (Cavalier-Smith, 1985). DNA is usually present in excess of the most generous estimates of genotypic requirements, often as untranscribed repetitive sequences. While variation in heterochromatic blocks of highly repetitive sequences is usually indicative of chromosomal polymorphisms (e.g. Arnold et al., 1986), moderately repetitive sequences exhibit different patterns of interspersion in euchromatin which accompany phylogenetic divergence (Mizuno \& Macgregor, 1974; Bouchard, 1982; Narayan, 1982). Extra DNA has variously been described as harmless junk (Ohno, 1985), parasitic (Orgel \& Crick, 1980) or of nucleotypic value (Cavalier-Smith, 1985). Studies of possible nucleotypic

*Present address: Department of Medical Genetics, St Mary's Hospital, Hathersage Road, Manchester M1 3 0JH, U.K.

†Correspondence.

This and the following paper are dedicated to the memory of our friend and colleague J. N. Hartshorne. effects have revealed that meiosis is much more sensitive to differences in genome size than is mitosis. In flowering plants, for instance, a 25 -fold increase in $2 \mathrm{C}$ DNA amount is accompanied by little more than a doubling of mitotic cycle time, whereas the duration of meiosis is extended by a factor of 10 (Rees \& Jones, 1977, p. 33).

A crucial yet enigmatic aspect to meiosis is the ability of homologous chromosomes to associate at zygotene. In theory, a minimum of one synaptic site per chromosome would be sufficient for pairwise synapsis, giving rise to bivalents at pachytene. Although multiple pairing initiations are commonly observed in plants, the characteristics of synaptic sites only become apparent under conditions of competitive pairwise synapsis and are therefore most readily studied in autopolyploids where each chromosome has more than one potential pairing partner. In the majority of polyploids, multivalents arise through the pairwise association of chromosomes at two or more autonomous synaptic sites (Moens, 1969; Loidl, 1986; Loidl \& Jones, 1986; Vincent, 1991). The autonomous nature of synaptic sites is exposed by their ability to generate changes of pairing partner in autopolyploids (Darlington, 1929). An autonomous synaptic site may be defined as the smallest region of a chromosome with the capacity to initiate changes in pairing partner in an autopolyploid (Hamey et al., 1988). 
If genomic evolution alters the number or spacing of autonomous synaptic sites, a mathematical model shows that such changes should have distinguishable effects on bivalent and multivalent frequency in polyploids (Callow \& Gladwell, 1980; 1984). While any synaptic effects are liable to be mitigated by selection in established polyploids (Callow \& Parker, 1985), they can be of little significance at the diploid level. Synaptic responses to concerted genomic evolution are therefore most likely to be detected in newly derived $\left(\mathrm{C}_{0}\right)$ autotetraploids.

Species of Lathyrus (Fabaceae) are ideal for nucleotypic studies of intergenomic divergence. They have a common basic number $(x=7)$, are usually diploid ( 88 per cent) (Darlington \& Wylie, 1955) but display extensive variation in genome size (7-29 pg DNA/2C) and consistent differences in genomic composition, particularly in relation to moderately repetitive sequences (Narayan, 1982; Kuriyan \& Narayan, 1988). The effects of such intergenomic divergence on the mechanics of pairing autonomy have been studied by comparing the meiotic behaviour of a series of $\mathrm{C}_{0}$ autotetraploids with the predictions of the model. The results are reported in this paper. Intragenomic comparisons are the subject of the sister paper in this series (Wallace \& Callow, 1993), while influences of ploidy and chiasma variation will be explored elsewhere.

The term 'pairing partner exchange' has long been applied to both the process of exchanging pairing partners and the structural intersections to which that process gives rise (e.g. Darlington, 1929; Rasmussen, 1987). We use 'synaptic exchange' in the same sense, only modifying the phrase for simplicity and to conform with the use of 'synaptic site' and 'synaptic interval' in our chosen mathematical model (Callow \& Gladwell, 1984; Hamey et al., 1988). As far as structures are concerned, some authors now prefer the term 'pairing partner switch' (e.g. Loidl \& Jones, 1986; Gillies et al., 1987).

\section{Model}

Observations of meiotic events in $\mathrm{C}_{0}$ autotetraploids have been tested against the predictions of a simple model which may be extended to include any type or degree of polyploidy (Callow \& Gladwell, 1980; 1984). Here, we are principally concerned with inter-relations between chromosome size, numbers of autonomous synaptic sites, sizes of synaptic intervals between sites and the incidence of synaptic exchange within such intervals. Because such aspects of the model are crucial to our evidence, pertinent details of the argument are outlined below.

\section{Assumptions}

1 Maximal pairwise association at zygotene; any univalents and trivalents observed at metaphase- 1 are thereby assumed to result from the desynaptic breakdown of bivalents or quadrivalents.

2 Pairing at an average of $i$ autonomous synaptic sites on each chromosome of a genome.

3 Each synaptic interval being of similar length, $\theta$, within a genome.

4 Each synaptic interval within a genome having the same probability, $\rho$, of including a single synaptic exchange (Fig. 1a and $b$ ).

5 The probability $\rho$ being effectively linearly related to the length of each synaptic interval $\theta$.

Assumption 5 only holds for small values of $\theta$ because $\rho$ is expected to approach the limit imposed by random pairing at large values of $\theta$.

\section{Derivations}

1 The probability, $\alpha$, of a set of four homologous chromosomes forming two bivalents at pachytene is the same as that of no synaptic exchange $(1-\rho)$ at each of $i-1$ synaptic intervals (Fig. 1c).

Thus we have

$\alpha=(1-\rho)^{i-1}$

on logarithmic transformation this yields

$\ln \alpha=(i-1) \quad \ln (1-\rho)$.

2 Each chromosome has an effective synaptic length, $\lambda$, which is the sum of all $i-1$ synaptic intervals, each of length $\theta$,

giving

$\lambda=(i-1) \theta$.

3 As the average number of synaptic exchanges $\bar{r}$ is $\rho(i-1)$, we have

$i-1=\bar{r} / \rho$

but from equation 2 ,

$i-1=\lambda / \theta$.

We now see that the average number of synaptic exchanges is expected to be linearly related to effective synaptic length, where $\theta$ and $\rho$ are constant because

$\bar{r}=(\rho / \theta) \lambda$.

4 Consistent differences in genome size may have three possible outcomes with regard to synaptic behaviour (Fig. 1d): (i) they may be unrelated to effective synaptic length, possibly contributing to chromatin loops on the periphery of synaptonemal 
complexes; (ii) they may alter the number of synaptic sites $i$ or (iii) they may change the size of synaptic intervals $\theta$. In the first case, pachytene bivalent frequency should be unrelated to genome size. In the second, it should bear a $\log /$ linear relation to chromosome size and, in the third a $\log / \log$ relation. These conclusions stem from $\alpha$ showing a $\log / \operatorname{linear}$ relation to $i$ but a $\log /$ $\log$ relation to $\rho$ (equation 1 ) whereas $\lambda$ is a linear function of both $i$ (equation 2) and $\rho$ (equation 2 and assumption 5).

\section{Materials and methods}

Seven Lathyrus spp. were obtained through the European Seed Exchange Scheme. $C_{0}$ autotetraploid plants

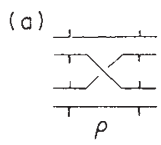

(b)
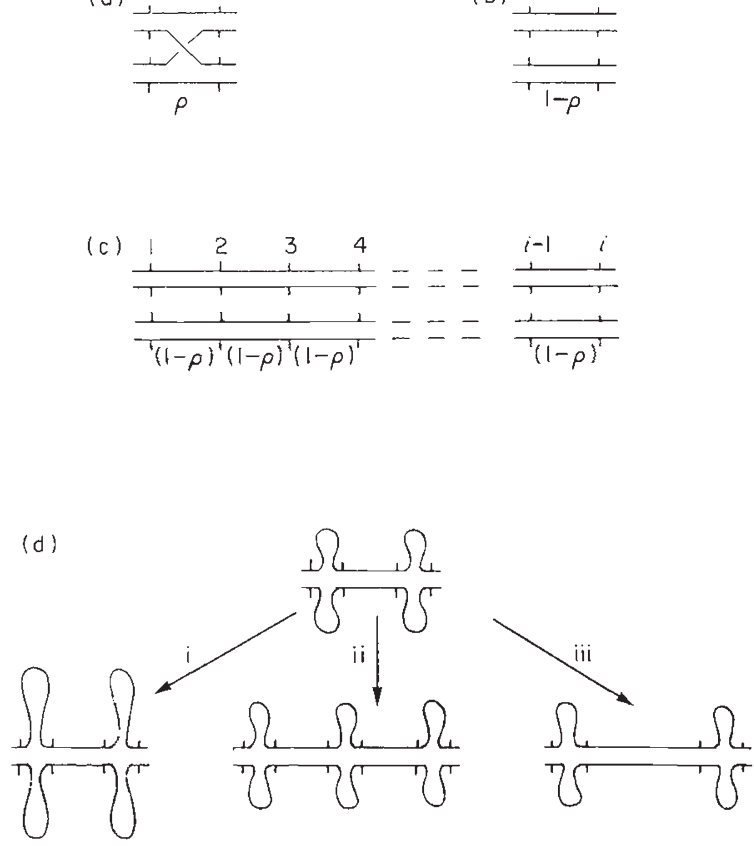

Fig. 1 Scheme of pairing at $i$ equidistant autonomous sites in four homologous chromosomes (see Model). Two mutually exclusive pairing events are assumed to occur with probabilities of $\rho$ and $1-\rho$ at each of the $i-1$ synaptic intervals: discordance between successive synaptic sites, producing synaptic exchange amongst all four homologues (a) and concordance, resulting in two paired segments (b). The probability, $\alpha$, with which a set of four homologous chromosomes is expected to form two bivalents at pachytene is therefore $(1-\rho)^{i-1}$ because no exchange must occur $(c)$. On logarithmic transformation, this yields $\ln \alpha=(i-1) \ln (1-\rho)$ (equation 1). Genomic increases arising from the interspersion of DNA sequences (d) may interact with effective synaptic length in three different ways: (i) by contributing only to the lateral loops of a synaptonemal complex they may make no contribution to effective synaptic length, (ii) they may increase the number of synaptic sites and (iii) they may expand synaptic intervals. were produced by immersing germinating seedlings in a 0.01 per cent colchicine solution for $12 \mathrm{~h}$. Seedlings were rinsed thoroughly under running water for $3 \mathrm{~h}$ before replanting in a peat-based compost. Surviving plants were grown until flowering in a glasshouse maintained at $20^{\circ} \mathrm{C}$ supplemented with light from sodium vapour lamps for $8 \mathrm{~h} /$ day. Suitable individuals for meiotic analysis were selected by examining fixed shoot-tip meristems for tetraploid cells.

Root- and shoot-tip squashes in lacto-propionic orcein were prepared after $3 \mathrm{~h}$ pre-treatment in 0.05 per cent colchicine, followed by fixation in ethanol/ acetic acid (3:1) and staining in Feulgen. Meiotic preparations from suitably staged anthers were prepared similarly but without the colchicine pre-treatment. Thirty-six plants were examined, at least three per species (Table 1).

Estimates of pachytene pairing behaviour are based on observations of pollen mother cells (PMCs) at metaphase-I. While direct observations of other polyploids have been made using pachytene spreading (e.g. Loidl \& Jones, 1986), the technique has yet to be adapted for Lathyrus and could not be expected to yield the large body of data required for our analysis.

Inferences of pachytene behaviour, drawn from observations of metaphase-I, are liable to be distorted by the repulsion of non-sister chromatids at diplotene, unless pairs of non-sisters are bound by chiasmata. Multivalents are particularly prone to such desynaptic disintegration (John \& Henderson, 1962; Roseweir \& Rees, 1962). As far as tetrasomic sets of homologous chromosomes (tetrasomes) are concerned, at least three chiasmata are required to maintain a quadrivalent to metaphase-I, whereas only two chiasmata are needed to maintain the same number of chromosomes as bivalents. Cells with seven tetrasomes therefore require at least 21 chiasmata to maintain the maximum of seven quadrivalents to metaphase-I. To increase the likelihood that each tetrasome within the cell forms at least three chiasmata, PMCs of Lathyrus were selected, each with a minimum of 28 chiasmata and hence a mean of at least four chiasmata per tetrasome. This should minimize lapses of pachytene pairing due to insufficient chiasmata.

Although the ensuing sample sizes varied according to the original chiasma frequency (Table 1), a large number of cells (942) was investigated with a mean very close to the selected minimum of 28 chiasmata (30.6). Studies of relations between metaphase-I pairing behaviour and chiasma frequency in the full sample of PMCs are to be published elsewhere. They indicate that the present selection of cells of high chiasma frequency does not appreciably bias the estimated range of pachytene behaviour under scrutiny. Thus, extra- 
polations from the full spectrum of chiasma variation give similar estimates of pairing behaviour at high chiasma frequency to those reported here for the selected samples. Because our interest was in the presence or absence of synaptic exchange, pairs of univalents were counted as bivalents whereas single univalents accompanied by trivalents were counted as quadrivalents. Both categories were rare (Table 1).

Inferences on the incidence of synaptic exchange are especially susceptible to both chiasma frequency and chiasma distribution because each synaptic exchange will only persist to metaphase-I if individually flanked by chiasmate associations. We are therefore only able to infer minimal numbers of synaptic exchanges from our observations.

DNA amounts are those reported by Narayan (1982). Relations between bivalent frequency and DNA amount are tested in a series of regression analyses. Partial regression has been employed to detect the most critical genomic component, followed by curvilinear regression to distinguish between possibilities 4(ii) and 4(iii) in the above model.

As Narayan \& Rees (1976) have pointed out, variation in the size of the genome amongst Lathyrus species is largely attributable to the size of the moderately repetitive component. The seven species investigated here bear testimony to this conclusion. Using the values reported by Narayan (1982), we see that moderately repetitive DNA $(M)$ increases steeply with genome size $(1 C) \quad(\hat{M}=-3.513+0.795 C$, $\left.t_{[5]}=12.986, P<0.001\right)$, whereas non-repetitive DNA
$(N)$ increases only gradually $(\hat{N}=2.708+0.178 C$, $\left.t_{[5]}=3.054, P<0.05\right)$ and highly repetitive DNA $(H)$ shows no relation to genome size $(\hat{H}=0.805+0.028 C$, $\left.t_{[5]}=0.333, P>0.70\right)$.

\section{Results}

Observations of mitotic complements confirm previous reports of variation in chromosome size reflecting amounts of total genomic DNA (Narayan, 1982). The total mitotic length in Lathyrus hirsutus $(19.9 \mathrm{pg} / 2 \mathrm{C})$, for example, shows a 36 per cent increase over that of L. articulatus $(12.2 \mathrm{pg} / 2 \mathrm{C}$ ) (Fig. 2) (see Wallace \& Callow, 1993).

Although at least 100 cells per species were screened at metaphase-I, sample sizes of those with a minimum of 28 chiasmata varied between 29 and 391 in accordance with the degree of chiasma variation in each species (Table 1). Mean chiasma frequencies of the selected samples ranged over a very narrow band (29.0-32.4/PMC). Six of the seven species exhibited a limited spectrum of mean quadrivalent frequency (3.58-3.99/PMC). The remaining species, $L$. aphaca, formed on average one additional quadrivalent. In all cases univalents and trivalents were rare (Table 1) (Fig. 3).

The proportion $(\alpha)$ of tetrasomes, forming two bivalents at pachytene, is expected to attain a maximum value of one-third, under conditions of random association between homologous synaptic sites. This may be due to independent association at either end of one (a)

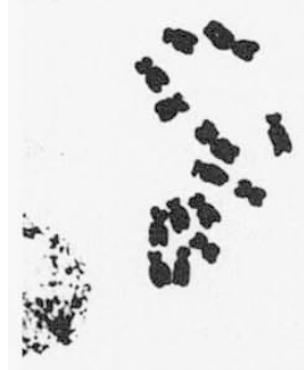

(c)

(b)

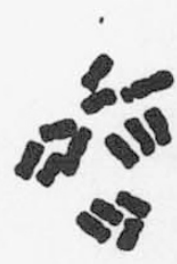

(d)

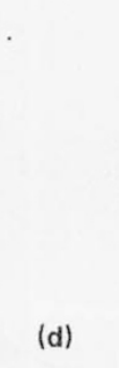

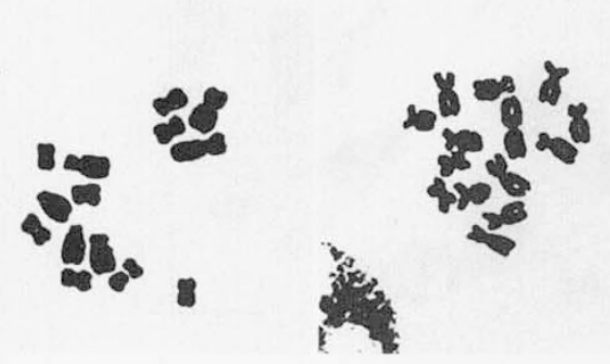

(e)

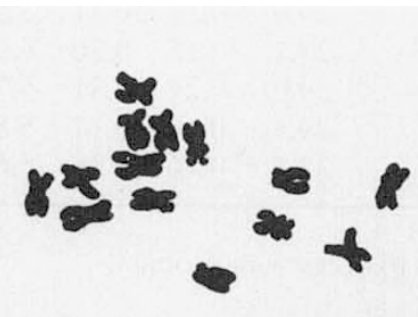

(f)

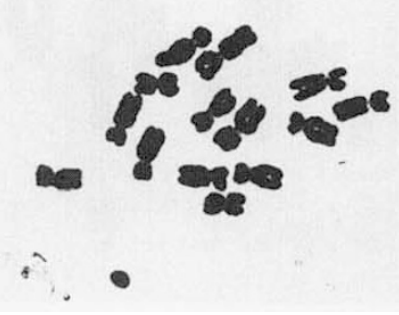

(g)

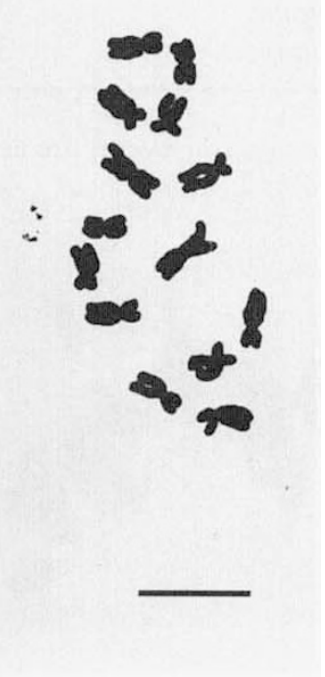

Fig. 2 The diploid complements of seven species of Lathyrus observed at mitotic metaphase. (a) L. angulatus, (b) L. articulatus, (c) L. aphaca, (d) L. cicera, (e) L. sativus, (f) L. odoratus, (g) L. hirsutus. Bar $=10 \mu \mathrm{m}$. 
large synaptic interval or to the equivalent collective behaviour of many synaptic sites (Callow \& Gladwell, 1984; Callow et al., 1984). The estimated value of $\alpha$ in $\mathrm{C}_{0}$ autotetraploid $L$. aphaca does not differ significantly from this prediction $(0.260$ 2IIs/PMC, $\left.t_{[99]}=1.591, P>0.05\right)$. By contrast, $\mathrm{C}_{0}$ autotetraploid $L$. hirsutus, with the next lowest estimate of $\alpha(0.405)$, shows an excess of bivalents and a highly significant deviation from random pairing $\left(t_{\mid 390]}=2.88, P<0.01\right)$.

With the exception of $L$. aphaca, estimates of $\alpha$ show a consistent decline with increasing genome size (Table 2). This decline is strictly log/linear and highly significant (Fig. 5a) (Table 3i), in accordance with the prediction of the model (4ii) where the number of synaptic sites changes with the size of the genome whereas the lengths of synaptic intervals remain constant. Partial regression analysis shows this relation to be attributable to the size of the moderately repetitive fraction (Table 3ii). Not only was the partial regression coefficient for moderately repetitive DNA the only coefficient to bear the expected negative sign but it was the only coefficient to prove significant. The logarithm of bivalent frequency shows a highly significant linear decline with increasing amounts of moderately repetitive DNA (Fig. 5b) and gives no indication of a nonlinear element (Table 3iii). These observations are consistent with the hypothesis that number of autonomous synaptic sites is sensitive to genome size (Model, 4ii) and particularly to the size of the moderately repetitive fraction.

Minimum numbers of synaptic exchanges were inferred from metaphase configurations of multivalents (Fig. 4). With the exception of a single quadrivalent in $L$. aphaca which required at least three synaptic exchanges, estimates of the minimum number of exchanges varied from one to two per multiple and from 0 to 10 per cell. The average minimum ranged from 3.73 synaptic exchanges per cell in $L$. angulatus to 5.52 in $L$. aphaca. These values represent a range of 0.532 to 0.789 per tetrasome (Table 2 ). By contrast, the minimum number of synaptic exchanges per multivalent remained close to unity in autotetraploids of all seven species (1.014-1.091), L. aphaca yielding an estimate close to the median (1.066) (Table 2).

Table 1 Average metaphase-I pairing behaviour in PMCs each with a minimum of 28 chiasmata sampled from $36 \mathrm{C}_{0}$ autotetraploids of seven species of Lathyrus

\begin{tabular}{|c|c|c|c|c|c|c|c|c|}
\hline \multirow[b]{2}{*}{ Species } & \multirow{2}{*}{$\begin{array}{l}\text { Number of } \\
\text { plants }\end{array}$} & \multirow{2}{*}{$\begin{array}{l}\text { Number of } \\
\text { PMCs }\end{array}$} & \multicolumn{2}{|c|}{$\begin{array}{l}\text { Chiasma } \\
\text { frequency }\end{array}$} & \multicolumn{4}{|c|}{ Mean pairing pattern } \\
\hline & & & Mean & S.E. & I & II & III & IV \\
\hline L. angulatus & 3 & 62 & 30.3 & 0.31 & 0.26 & 6.56 & 0.10 & 3.58 \\
\hline L. articulatus & 5 & 94 & 29.5 & 0.29 & 0.27 & 6.29 & 0.16 & 3.67 \\
\hline L. aphaca & 8 & 100 & 29.0 & 0.14 & 0.31 & 3.61 & 0.25 & 4.93 \\
\hline L. cicera & 4 & 54 & 29.1 & 0.15 & 0.20 & 6.26 & 0.13 & 3.72 \\
\hline L. sativus & 4 & 29 & 29.0 & 0.21 & 0.31 & 5.72 & 0.17 & 3.93 \\
\hline L. odoratus & 4 & 212 & 29.4 & 0.19 & 0.31 & 5.82 & 0.22 & 3.85 \\
\hline L. hirsutus & 8 & 391 & 32.4 & 0.16 & 0.29 & 5.61 & 0.17 & 3.99 \\
\hline
\end{tabular}

(a)

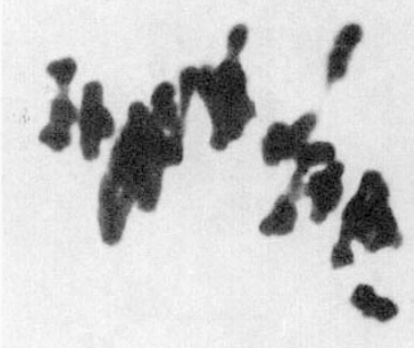

(b)

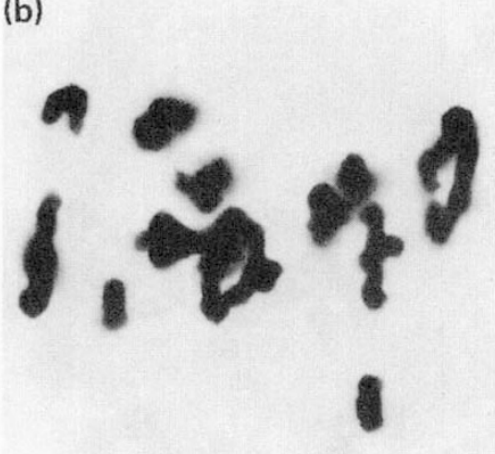

(c)

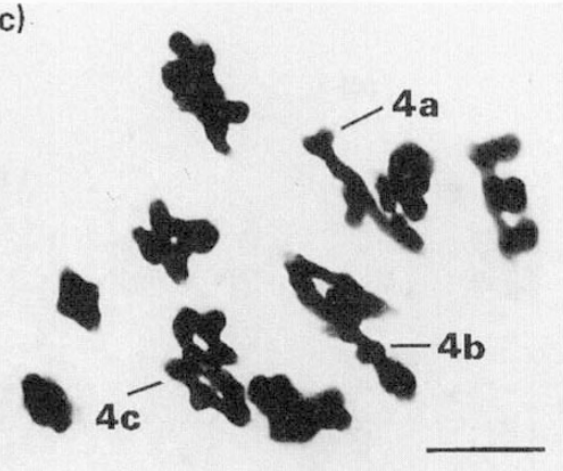

Fig. 3 Metaphases-I PMCs from $\mathrm{C}_{0}$ autotetraploids of three species of Lathyrus: (a) L. angulatus $\left(1_{\mathrm{I}} 6_{\mathrm{II}} 1_{\mathrm{III}} 3_{\mathrm{IV}}\right)$, (b) L. aphaca $\left(2_{\mathrm{I}}\right.$ $\left.10_{\mathrm{II}} 4_{\mathrm{IV}}\right)$ and $(\mathrm{c})$ L. hirsutus $\left(4_{\mathrm{II}} 5_{\mathrm{IV}}\right)$. The formation of the quadrivalents labelled $4 \mathrm{a}, 4 \mathrm{~b}$ and $4 \mathrm{c}$ is depicted in Fig. 4 . Bar $=10 \mu \mathrm{m}$. 
The incidence of minimal synaptic exchange increased significantly with the size of the genome, again excluding L. aphaca (Fig. 6a) (Tables 2, 3iv). Although none of the partial regression coefficients for the various DNA components proved significant, that for moderately repetitive DNA came closest and was the only coefficient to bear the appropriate positive sign (Table $3 \mathrm{v}$ ). Indeed, a separate regression against the size of the moderately repetitive component by itself proved significant (Table 3vi) (Fig. 6b). This final

Table 2 Proportions $(\alpha)$ of tetrasomes forming two bivalents at pachytene and average minimal numbers of synaptic exchanges $(\bar{r})$ per tetrasome, estimated from $\mathrm{C}_{0}$ autotetraploid Lathyrus PMCs each forming at least 28 chiasmata and compared with estimates of genomic composition

\begin{tabular}{|c|c|c|c|c|c|c|c|c|c|}
\hline \multirow[b]{2}{*}{ Species } & \multicolumn{4}{|c|}{$\begin{array}{l}\text { DNA composition* } \\
\text { pg/unreplicated } \\
\text { chromosome }\end{array}$} & \multicolumn{2}{|c|}{$\begin{array}{l}\text { Bivalent } \\
\text { frequency }\end{array}$} & \multicolumn{2}{|c|}{$\begin{array}{l}\text { Synaptic } \\
\text { exchange }\end{array}$} & \multirow[b]{2}{*}{$\bar{r} /$ mult. } \\
\hline & Non & Mod. & High & Total & $\alpha$ & $s_{\alpha}$ & $\bar{r}$ & $s_{\mathrm{r}}$ & \\
\hline L. angulatus & 0.31 & 0.35 & 0.11 & 0.77 & 0.475 & 0.063 & 0.532 & 0.065 & 1.014 \\
\hline L. articulatus & 0.37 & 0.47 & 0.03 & 0.87 & 0.453 & 0.051 & 0.570 & 0.056 & 1.042 \\
\hline L. aphaca & 0.36 & 0.50 & 0.14 & 1.00 & 0.260 & 0.044 & 0.789 & 0.052 & 1.066 \\
\hline L. cicera & 0.41 & 0.55 & 0.04 & 1.00 & 0.450 & 0.068 & 0.601 & 0.079 & 1.091 \\
\hline L. sativus & 0.36 & 0.74 & 0.10 & 1.20 & 0.414 & 0.091 & 0.635 & 0.107 & 1.083 \\
\hline L. odoratus & 0.41 & 0.70 & 0.10 & 1.21 & 0.419 & 0.034 & 0.628 & 0.039 & 1.079 \\
\hline L. hirsutus & 0.46 & 0.87 & 0.09 & 1.42 & 0.405 & 0.025 & 0.612 & 0.026 & 1.029 \\
\hline
\end{tabular}

*After Narayan (1982).

Table 3 Multiple regression analyses of relations between average meiotic pairing at high chiasma frequency and genomic composition in $C_{0}$ autotetraploids representing six species of Lathyrus: $L$. angulatus, $L$. articulatus, $L$. cicera, $L$. odoratus, $L$. sativus and $L$. hirsutus. These regressions test predictions of the pairing model (see Fig. 1). DNA amounts are in pg/unreplicated chromosome (see Table 2)

(i) Effect of total DNA on bivalent frequency $\ln \alpha=-0.561-0.251$ total DNA; $t_{[4]}=-8.463 ; \quad P<0.01$ (deviation from linearity, $F_{[1 / 3]}=0.974, P>0.05$ )

(ii) Effects of DNA fractions on bivalent frequency $\ln a=-0.701+0.242$ non rep. -0.376 mod. rep. +0.080 high rep.

$$
\begin{array}{lll}
t_{[2]}=1.070 & t_{[2]}=-6.059 & t_{[2]}=0.363 \\
P>0.30 & P<0.05 & P>0.70
\end{array}
$$

(iii) Effect of moderately repetitive DNA on bivalent frequency $\ln \alpha=-0.635-0.321$ mod. rep. DNA; $t_{[4]}=-12.267 ; \quad P<0.01$ (deviation from linearity, $\mathrm{F}_{[1 / 3]}=0.907, P>0.05$ )

(iv) Effect of total DNA on minimal synaptic exchange $\bar{r}=0.452+0.134$ total DNA; $t_{[4]}=2.974 ; \quad P<0.05$

(v) Effects of DNA fractions on minimal synaptic exchange $\bar{r}=0.585-0.307$ non. rep. +0.255 mod. rep. -0.366 high rep

$$
\begin{array}{lll}
t_{[2]}=-0.586 & t_{[2]}=1.788 & t_{[2]}=-0.656 \\
P>0.30 & P>0.10 & P>0.30
\end{array}
$$

(vi) Effect of moderately repetitive DNA on minimal synaptic exchange $\bar{r}=0.489+0.175$ mod. rep. DNA $; t_{[4]}=3.366 ; \quad P<0.05$ 

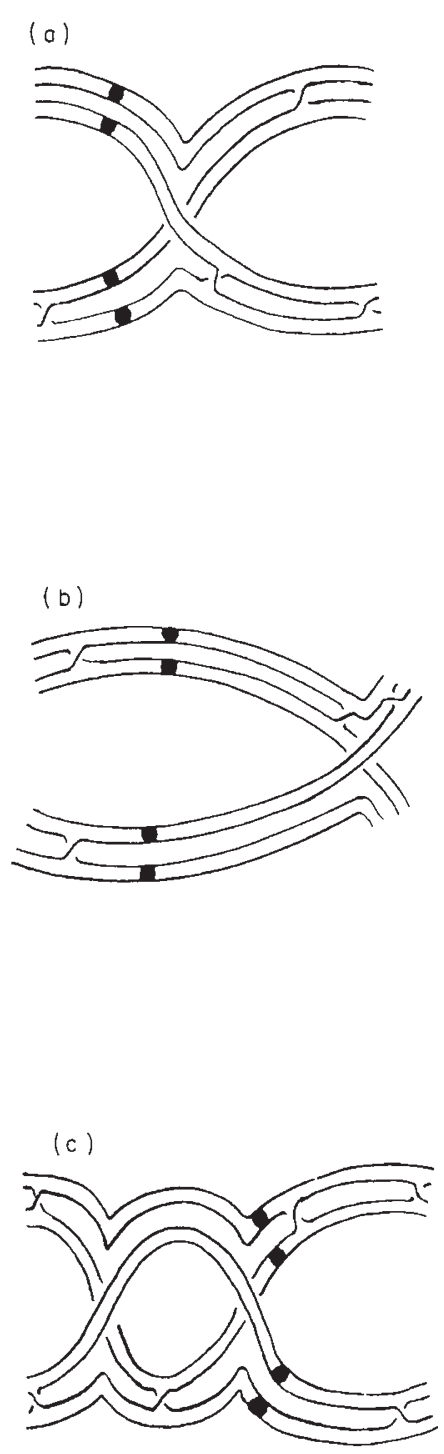

Fig. 4 Pachytene multiples inferred from the metaphase configurations of three quadrivalents in $\mathrm{C}_{0}$ autotetraploid $L$. hirsutus (see Fig. 3c). These quadrivalents give evidence of (a) a single non-localized synaptic exchange, (b) a single synaptic exchange localized between two distal chiasmata and (c) two synaptic exchanges within the same chromosome arm. Bar $=5 \mu \mathrm{m}$. Pachytene configurations are not drawn to scale.

relation concords with equation 3 and implies that amounts of moderately repetitive DNA are correlated with differences in effective synaptic length. Estimates of minimal numbers of synaptic exchanges and any analyses on which such estimates are based remain of limited value. A direct study based on pachytene spreads could provide sufficient data for measurement of the relation between the total incidence of synaptic exchange and the size of the genome. Such evidence would provide a useful test of the validity of assumption 5 .

\section{Discussion}

Chromosomal synapsis must represent the culmination of a complex hierarchy of molecular and structural interactions. At the molecular level, autonomous synaptic sites may be represented by interspersed specialized sequences consisting partly of conserved functional repeats and partly of unique regions of high pairing specifity. Alternatively, any random segment of genomic DNA may have the potential to pair autonomously. The possibility of specialized sequences is supported by the present paper, which implicates a moderately repetitive fraction in pairing autonomy. The nature of such sequences remains unknown. Some indication may be afforded by the findings of Stern \& Hotta (1987) but corroborative evidence is lacking. Observations of post-meiotic segregation and gene conversion support the concept of intimate pairing between non-sister coding sequences, leading to the formation of heteroduplexes (Hastings, 1987). This may follow the association of any autonomous synaptic sites and may even take place between chromatin loops outside the synaptonemal complex.

At the structural level, only up to about 0.3 per cent of the genome can be accounted for in the lateral elements of synaptonemal complexes (Lie \& Laane, 1982; Jones et al., 1983; John, 1990). Most of the chromatin is present as a series of uncomplexed loops (von Wettstein, et al., 1984). Synapsis in plant meiocytes begins at numerous independent chromosomal locations and the formation of synaptonemal complex is mediated by distinct pairing nodules (Jones \& Albini, 1988).

The autonomous nature of synaptic sites is revealed by synaptic exchange in polyploids (Darlington, 1929; Hamey et al., 1988). The reported incidence of synaptic exchange in triploid Allium sphaerocephalon (Loidl \& Jones, 1986) and tetraploid A. vineale (Loidl, 1986) has been shown to follow a Poisson frequency distribution (Hamey et al., 1988). A similar analysis has confirmed that synaptic exchanges show Poisson frequency distributions in autopolyploid Crepis capillaris (J. E. Vincent \& G. H. Jones, personal communication). In all these cases, therefore, we have evidence of a rare event being equally likely at a large number of stations. The implication of such evidence is of large numbers of synaptic intervals, each with a low probability of synaptic exchange (Hamey et al., 1988). Under such conditions, synapsis will be quasi-continuous (Callow \& Gladwell, 1984; Hamey et al., 1988).

Concerted reductions in genome size during the evolution of Lathyrus have been accompanied by loss 
Fig. 5 Relations between the natural logarithm of the bivalent frequency $(\ln \alpha),(a)$ total genomic DNA content and (b) moderately repetitive DNA content in seven Lathyrus species (see Table 3). Samples are based on PMCs with a minimum number of 28 chiasmata. Regression analysis does not include $L$. aphaca. (A) L. angulatus, (B) L. articulatus, (C) L. aphaca, (D) L. cicera, (E) L. sativus, (F) L. odoratus, (G) L. hirsutus.
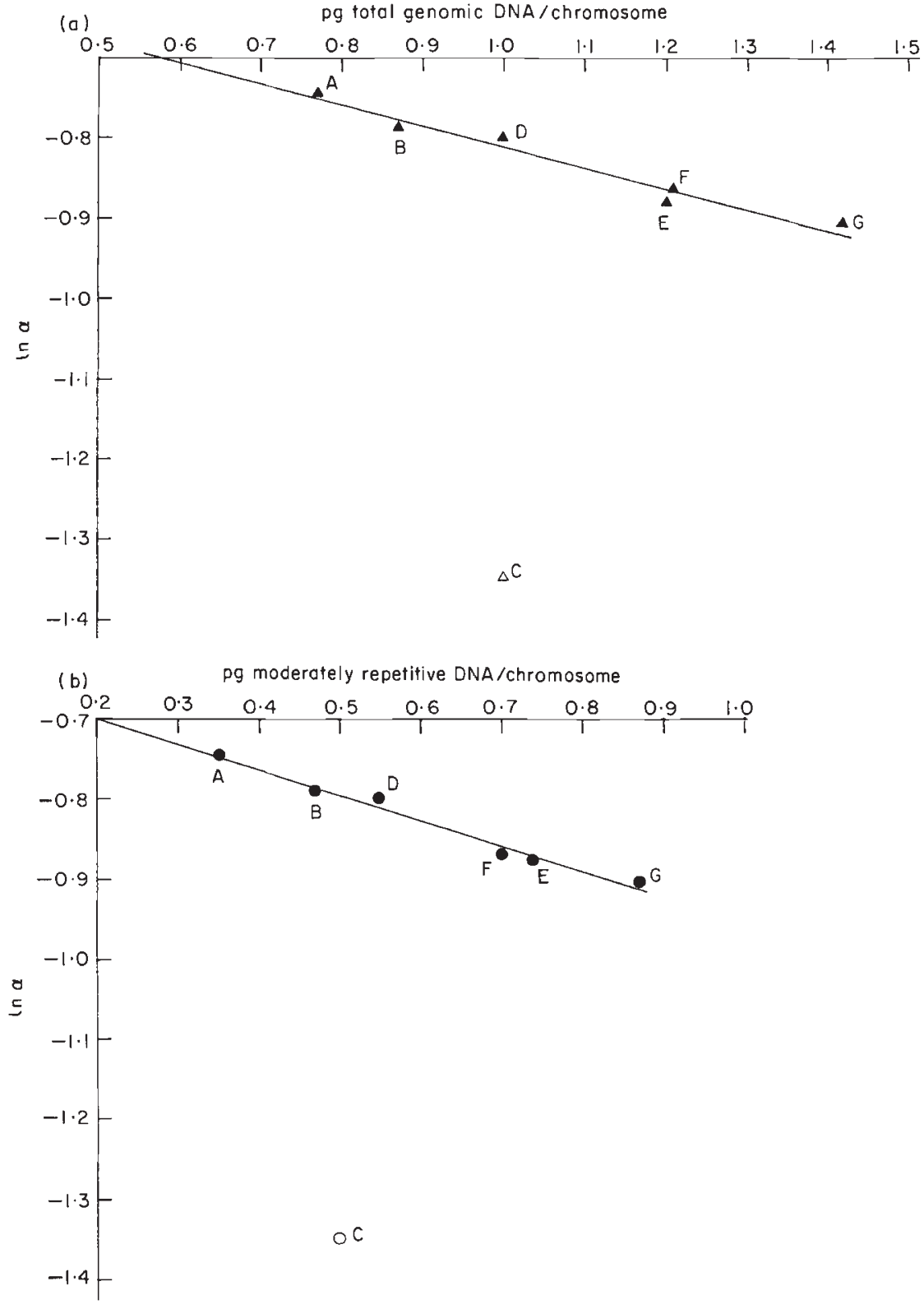

of some non-repetitive DNA but have been achieved largely at the expense of moderately repetitive material (Narayan, 1982; Kuriyan \& Narayan, 1988). Six of the seven species investigated here constitute a nucleotypic series, within which the number of autonomous synaptic sites declines with genome size but the spacing of such sites remains constant. The extent to which the members of this series represent an evolutionary progression, remains unknown.

Three features of our analyses point to the moderately repetitive genomic component as the source of autonomous synaptic sites. Firstly, although non-repetitive and moderately repetitive DNA are both significant correlates of genomic size in Lathyrus
(Narayan, 1982), only the latter genomic component yields a significant decline in bivalent frequency. The partial regression coefficient relating bivalent frequency to the size of the non-repetitive component is not only non-significant but positive. Secondly, the six species of the nucleotypic series give identical rankings for bivalent frequency and the amount of moderately repetitive DNA but not for genome size. Thirdly, only the size of the moderately repetitive genomic component yields the predicted positive relation with the average minimum number of synaptic exchanges.

Should autonomous synaptic sites reside in the moderately repetitive component, they could be interspersed throughout the genome and yet include core 


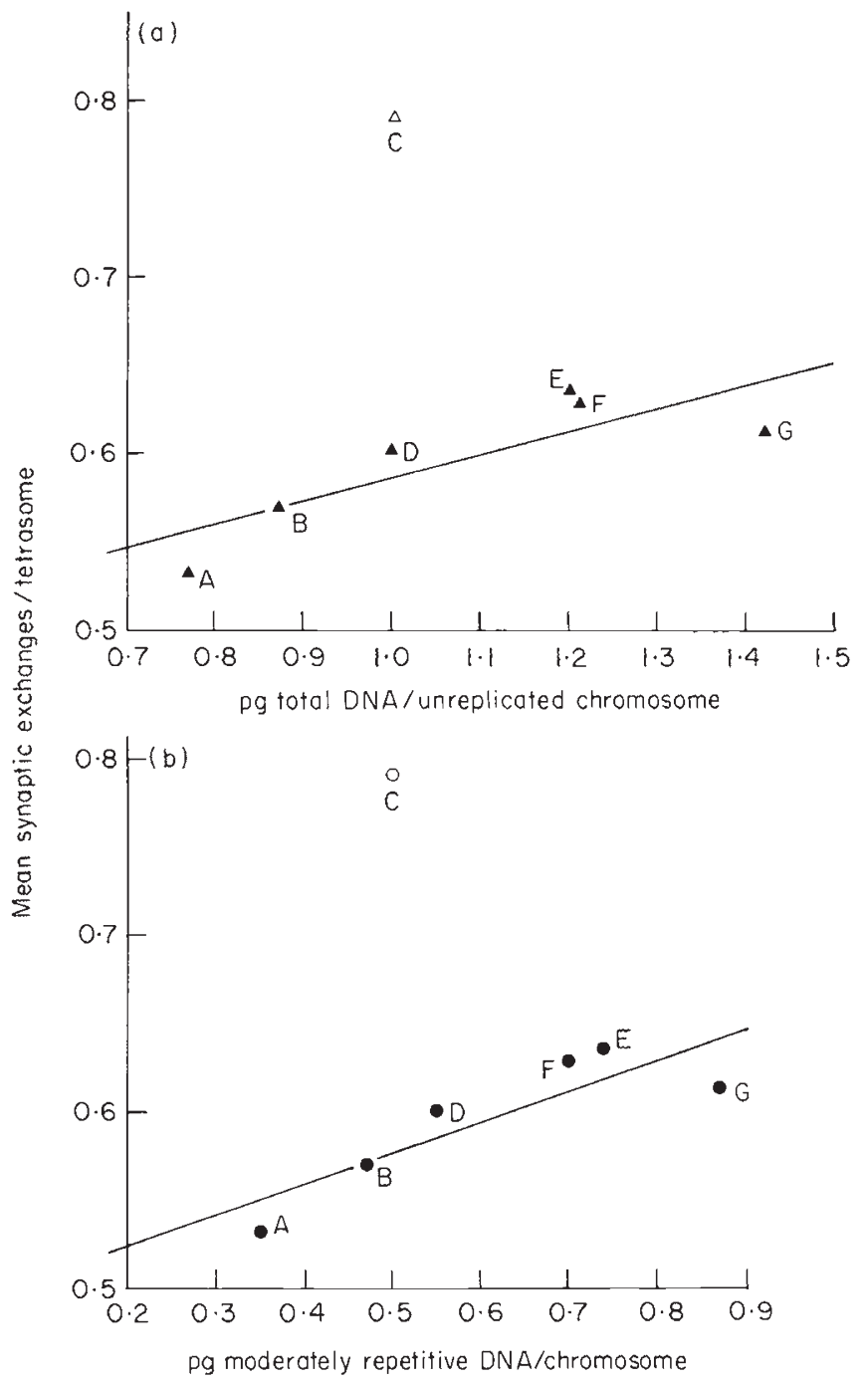

Fig. 6 Relations between the average incidence of synaptic exchange $(\bar{r}),(a)$ total genomic DNA content and (b) moderately repetitive DNA content in seven Lathyrus species (see Table 3). Samples are based on PMCs with a minimum number of 28 chiasmata. Regression analysis does not include L. aphaca. Species codes are as in Fig. 5.

sequences of common function. Synaptic sequences may or may not be transcribed because pairing initiation could conceivably occur without transcription.

Although the taxonomy of Lathyrus species is in need of review, such treatments that exist all place $L$. aphaca in a distinct subsection of the genus (Senn, 1938; Davis, 1970). Our finding that this species has a distinctive pattern of meiotic behaviour therefore accords with the possibility of a separate phyletic origin. Indeed, a genus as large as Lathyrus is very likely to be represented by several nucleotypic series of the type reported here. While we can as yet have no inkling of the mechanical nature of any differences between the nucleotypic series, some pointers are available from our data. Despite their variation in quadrivalent frequency, $\mathrm{C}_{0}$ autotetraploids of all seven species gave very similar estimates of the minimum number of synaptic exchanges per multivalent. Even Lathyrus aphaca with its distinctively high quadrivalent frequency showed only an average minimum of one synaptic exchange per multiple. Apparently, therefore, the genome of $L$. aphaca is neither especially rich in autonomous synaptic sites nor does it appear likely to contain unusually large synaptic intervals. The evidence of random association in this species may, however, point to a high level of homozygosity for pairing sequences (Callow et al., 1984).

Previous intergenomic comparisons of Lathyrus species have revealed no evidence of a nucleotypic relation between chiasma frequency and genome size (Rees \& Durrant, 1986). Such comparisons are, however, liable to be confounded by influences of selection at the diploid level, particularly as reductions in genome size have been accompanied by transitions from outbreeding to inbreeding (Rees \& Dale, 1974). Changes in pairing autonomy are, by contrast, unlikely to be affected by selection, being detected in newly synthesized autotetraploids and of no adaptive significance to the original diploids.

\section{Acknowledgements}

We are very grateful to Dr G. H. Jones for his helpful comments on the manuscript. AJW thanks SERC for financial support.

\section{References}

ARNOLD, M. L., APPELS, R. AND SHAW, D. D. 1986. The heterochromatin of grasshoppers from the-Caledia captiva species complex. I. Sequence evolution and conservation in a highly repeated DNA family. Mol. Biol. Evol., 3, 29-43.

BOUCHARD, R. A. 1982. Moderately repetitive DNA in evolution. Int. Rev. Cytol., 76, 113-193.

CAllow, R. S. AND GLADWELL, I. 1980. Chromosome synapsis in hexaploids. J. Theor. Biol., 87, 703-722.

CALLOW, R. S. AND GLADWELL, I. 1984. A general treatment of chromosome synapsis in even-numbered polyploids. $J$. Theor. Biol., 106, 455-494.

CAllow, R. S., HAMEY, Y. AND PATTRICK, s. M. 1984. Pairing of identical chromosomes in an isogenic tetraploid. Heredity 53, 107-111.

CALLOW, R. S. AND PARKER, J. S. 1985. Hierarchical control of multivalent formation in natural populations of a hexaploid perennial grass (Koeleria vallesiana). Proc. $R$. Soc. Lond. B., 223, 459-473.

CAVALIER-SMITH, T. 1985. Eukaryote gene numbers, noncoding DNA and genome size. In: Cavalier-Smith, T. (ed.) 
The Evolution of Genome Size. Wiley. Chichester, pp. $1-36$.

DARLington, C. D. 1929. The significance of chromosome behaviour in polyploids for the theory of meiosis. In: Conference on Polyploidy. John Innes Horticultural Institute, Merton, pp. 42-44.

DARLINGTON, C. D. AND WYLIE, A. P. 1955. Chromosome Atlas of Flowering Plants. George Allen and Unwin, London, pp. $155-156$.

DAVIS, P. H. 1970. Flora of Turkey, Volume 3. Edinburgh University Press, Edinburgh, pp. 328-369.

GILlIES, C. B., KUSPIRA, S. AND BHAMBHANI, R. N. 1987. Genetic and cytogenetic analyses of the A genome of Triticum monococcum. IV. Synaptonemal complex formation in autotetraploids. Genome, 29, 309-318.

HAMEY, Y., ABBERTON, M. T., WALLACE, A. J. AND CALLOW, R. S. 1988. Pairing autonomy and chromosome size. In: Brandham, P. E. (ed.) Kew Chromosome Conference III. HMSO, London, pp. 241-251.

HASTINGS, P. J. 1987. Meiotic recombination interpreted as heteroduplex correction. In: Moens, P. B. (ed.) Meiosis. Academic Press, Orlando, FL, pp. 107-137.

JoHN, B. 1990. Meiosis. Cambridge University Press, Cambridge, UK.

JOHN, B. AND HENDERSON, S. A. 1962. Asynapsis and polyploidy in Schistocerca paranensis. Chromosoma, 13, 111-147.

JONES, G. H. AND ALBINI, S. M. 1988. Meiotic roles of nodule structures in zygotene and pachytene nuclei of Angiosperms. In: Brandham, P. E. (ed.) Kew Chromosome Conference III. HMSO, London, pp. 323-330.

JONES, G. H., CROFT, J. A. AND WALlACE, B. M. N. 1983. Synaptonemal complexes in surface spread preparations of orthopteran spermatocytes. In: Brandham, P. E. and Bennett, M. D. (eds) Kew Chromosome Conference II. George Allen and Unwin, London, pp. 123-130.

KURIYAN, P. N. AND NARAYAN, R. K. J. 1988. The distribution and divergence during evolution of families of repetitive DNA sequences in Lathyrus species. J. Mol. Evol., 27, 303-310.

LIE, T. AND LAANE, M. M. 1982. Reconstruction analyses of synaptonemal complexes in haploid and diploid pachytene nuclei of Physarum polycephalum (Myxomycetes). Hereditas, 96, 119-140.

LOIDL, J. 1986. Synaptonemal complex spreading in $A$. vineale. Can. J. Genet. Cytol., 28, 754-761.
LOIDL, J. AND JONES, G. H. 1986. Synaptonemal complex spreading in Allium. I. Triploid A. sphaerocephalon. Chromosoma, 93, 420-428.

Mizuno, S. AND MACGREGor, H. C. 1974. Chromosomes, DNA sequences and evolution in salamanders of the genus Plethodon. Chromosoma, 48, 239-296.

MOENS, P. B. 1969. The fine structure of meiotic chromosome pairing in the triploid Lilium tigrinum. J. Cell Biol., 40 , 273-279.

NARAYAN, R. K. J. 1982. Discontinuous DNA variation in the evolution of plant species: the genus Lathyrus. Evolution, 36, 877-891.

NARAYAN, R. K. J. AND REES, H. 1976. Nuclear DNA variation in Lathyrus. Chromosoma, 54, 141-154.

oHNo, s. 1985. Dispensable genes. Trends Genet., 1, 160-164.

ORGEL, L. E. AND CRICK, F. H. C. 1980 . Selfish DNA: the ultimate parasite. Nature, 284, 604-607.

RASMUSSEN, S. W. 1987. Chromosome pairing in autotetraploid Bombyx males. Inhibition of multivalent correction by crossing over. Carlsberg Res. Commun., 52, 211-242.

REES, H. AND DALE, P. J. 1974. Chiasmata and variability in Lolium and Festuca populations. Chromosoma, 47, 335-351.

REES, H. AND DURRANT, A. 1986. Recombination and genome size. Theor. Appl. Genet., 73, 72-76.

REES, H. AND JONES, R. N. 1977. Chromosome Genetics. Edward Arnold, London.

ROSEWEIR, J. AND REES, H. 1962. Fertility and chromosome pairing in autotetraploid rye. Nature, 195, 203-204.

SENN, H. A. 1938. Experimental data for the revision of the genus Lathyrus. Am. J. Bot., 25, 67-78.

STERN, H. AND HOTTA, Y. 1987. The biochemistry of meiosis. In: Moens, P. B. (ed.) Meiosis. Academic Press, Orlando, FL, pp. 303-331.

vinCENT, J. E. 1991. Chromosome pairing in Crepis rubra and Crepis capillaris. Ph.D. Thesis, University of Birmingham.

WALlACE, A. J. AND CALlow, R. S. 1993. Synaptic responses to concerted genomic evolution in Lathyrus. II. Intragenomic effects. Heredity, 70, 92-97.

WETTSTEIN, D. VON, RASMUSSEN, S. W. AND HOLM, P. B. 1984. The synaptonemal complex in genetic segregation. Ann. Rev. Genet., 18, 331-413. 